\section{HYPOPLASIA OF LUNG WITH CONGENITAL DIAPHRAGMATIC HERNIA}

BY

\section{W. AREECHON,* M.B.}

AND

LYNNE REID, M.B., M.R.C.P., M.R.A.C.P.

From the Brompton Hospital and Institute of Diseases of the Chest

Hypoplasia of the lung - the term applied to subnormal lung volume-is commonly associated with conditions which affect the volume of the thorax available to the lung. Diaphragmatic hernia encroaches on and therefore reduces it; anencephaly and spina bifida impair the development of the ribs. Polycystic kidneys and renal agenesis may be associated with hypoplastic lung, though the nature of the relationship is less obvious.

Potter (1962) stated that with moderate degrees of hypoplasia the foetal lung is less mature than might normally be expected, and that the bronchi are disproportionately numerous, the alveoli and alveolar ducts being reduced in number. She did not specifically count bronchial generations. Campanale and Rowland (1955) describe the necropsy findings in five cases of congenital diaphragmatic hernia in which there was hypoplasia of the lung, and mention the same structural disparities as does Potter. In three of the five cases operation was performed. within 45 minutes, two hours, and 21 hours of birth, respectively and in each case it was found that on the side of the hernia the lung did not inflate properly.

Similar behaviour of hypoplastic lungs on inflation at operation had been mentioned by Barrett and Wheaton (1934) among others who have operated in this condition, but they also described operation at four and a half months on a child who survived for 17 days in whom at necropsy it was found that the affected lung had expanded "almost to normal." Tolins (1953) also reported a case operated upon at 22 hours, when attempts to reinflate the lung failed, so that immediately after the operation a pneumothorax was present, the lung being airless until the sixth day. when a large amount of thick mucus was aspirated at bronchoscopy. After this the lung gradually aerated and at 19 months the radiograph was normal. No investigation of the functioning of these lungs subsequent to operation has been reported.

In order to establish the effect of diaphragmatic hernia on the lung and the pulmonary structure on which the effect is greatest, the number of bronchial generations (or orders of branching) and the depth of the subpleural alveolar layer in both lungs of two foetuses have been investigated. Recently the antenatal development of the lung has been described (Bucher and Reid, 1961a, $196 \mathrm{lb})$ at different states of gestation by reference to the number of bronchial generations and the level to which cartilage and glands have extended, and these results have been used as the criteria of normal development.

\footnotetext{
* Present address, Central Chest Hospital, Bangkok, Thailand
}

\section{Material}

The first of the two patients studied was born at term, the second at the thirty-fourth week of intrauterine development. In the premature baby the opening in the diaphragm was smaller and less of the abdominal content was found in the thorax than in the case of the mature baby, in which the deficiency in the diaphragm was extensive.

Case 1.-Baby A. was a full-time male infant who had lived one hour: weight $3.580 \mathrm{~g}$. The necropsy report of Dr. W. Spector (University College Medical School) contained the following: "The mouth, pharynx, larynx, trachea, and main bronchi were normal with clear airway. The left side of the chest was filled with the intestines, spleen. stomach, and pancreas as the result of a large diaphragmatic hernia, the left half of the diaphragm being represented by a muscular band forming the anterior border of the defect. The heart was pushed to the right but otherwise appeared normal. The liver was deformed. part of the left lobe being present in the left half of the chest. The volume of the right lung was smaller than normal; the left lung was very much smaller and was represented by two flattened lobes each about the size of a thumb nail." From their arrangement and shape it was not possible on external examination to be certain which was which. It seemed likely, on cutting along the left main bronchus, that the first branch would pass to the upper lobe, which was the smaller of the two and triangular in shape. the lower being quadrilateral. The cranio-caudal length of the right lung was $7.3 \mathrm{~cm}$., and its maximum transverse diameter $5.6 \mathrm{~cm}$. Because of the distorted arrangement the measurements in the case of the left lung are not comparable, but the longest axis of one lobe was $2.4 \mathrm{~cm}$., that of the other $2.6 \mathrm{~cm}$.

Case 2.-Baby B. was a premature female infant, weight $2.110 \mathrm{~g}$., crown-heel length $430 \mathrm{~mm}$., crown-rump length $310 \mathrm{~mm}$., corresponding to 34 weeks' gestation; the child lived for four hours. The tongue, larynx, and trachea were normal. The length from larynx to carina was $4 \mathrm{~cm}$. and the airway in between was clear. The left side of the chest was occupied by the spleen and the intestine with the exception of the descending colon. these having gained entry by way of a left-sided defect, about 1 in. $(2.5 \mathrm{~cm}$.) across in the centre portion of the diaphragm, with broad bands of muscle at all borders of the defect. The right lung consisted of three lobes (Fig. 1), was voluminous, and was dark in colour. The left lung was very small, about one-fourth of the size of the right-with a relatively smooth but very pale surface; the upper lobe was the bigger and was triangular in shape. but the lower was small and flattened like a pancake. The cranio-caudal length of the right lung was $6 \mathrm{~cm}$. and the maximum anterior-posterior diameter was $4 \mathrm{~cm}$. Because of the distortion of the left lung comparable measurements could not be made. The maximum thickness of the lung was $2.5 \mathrm{~cm}$. for the right and $1 \mathrm{~cm}$. for the left.

\section{Methods}

As in both cases the left lung showed great reduction in size as well as distortion of shape: the whole lobe could be embedded in one block for sectioning. The plane of section was at right angles to the long axis of the main bronchus. Sections were cut at $5 \mu$ thickness and stained with haematoxylin-eosin, additional stains to identify cartilage and mucous glands being used on selected sections. The hilar region in Case I was mapped in detail on graph paper so as to gain an idea of bronchial segmental branching. All sections of the left lung in Case 1 were mounted, but the frequency of staining them varied-over the proximal regions sections were stained at $0.5-\mathrm{mm}$. intervals, then $0.25 \mathrm{~mm}$., and at the periphery every section. As pathways were traced in different segments extra sections could be stained as 
required. In Case 2 the same plan was followed save that in the hilar region sections were examined only at 1-mm. intervals (Fig. 2).

In Case 1 the whole of the right middle lobe of the right lung was sectioned as well as the anterior segment of the upper lobe and the posterior basal segment of the lower lobe. In Case 2, however, under the dissecting microscope the selected pathways were traced for some distance and their branches counted: only the peripheral region was sectioned; the medial segment of the middle lobe and the anterior segment of the upper lobe were examined in this way.

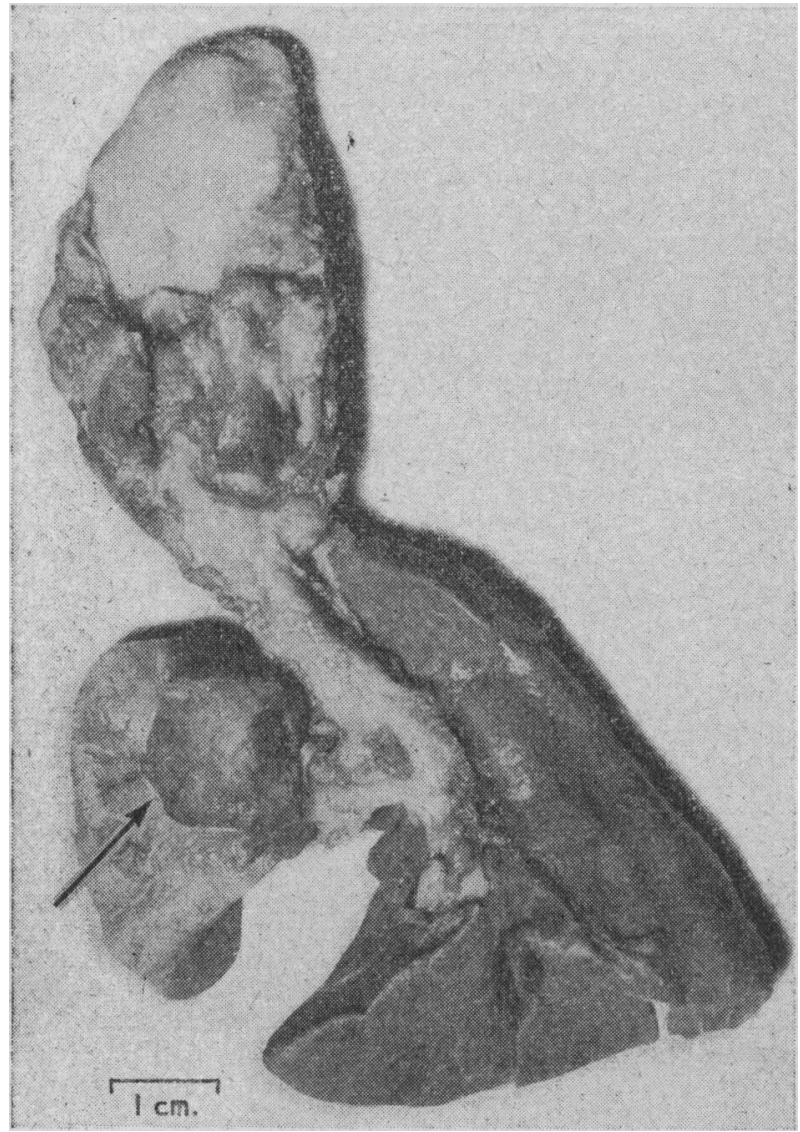

Fig. 1.-Case 2. Posterior view of lungs. The smaller, left, lung shows the lower lobe, its edge marked by an arrow, as a nub of tissue against the larger upper lobe: the right lung also is small compared with the tongue size.

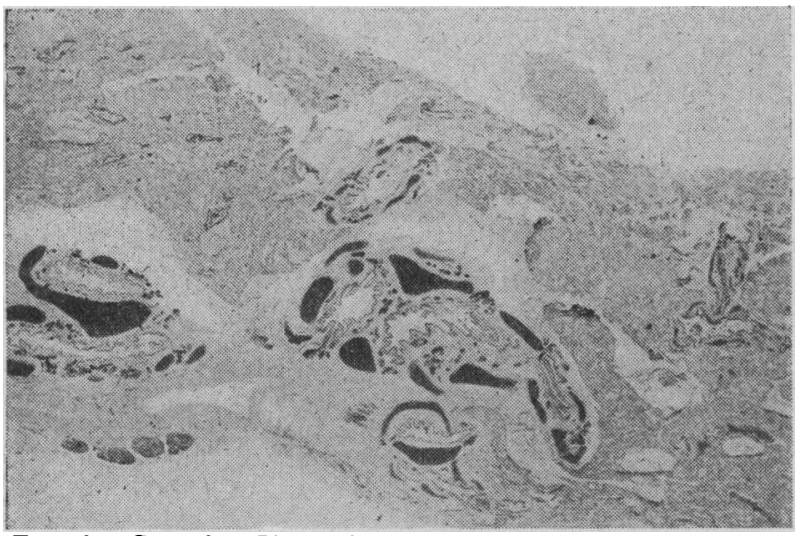

FIo. 2.-Case 2. Photomicrograph near hilum of left lung. Bronchi at hilum seem well developed, the plates of cartilage and glands appearing blick. As in a normal lobe, the peripheral plates of cartilage stain paler. A single section gives no idea of the degree of interference with the bronchial-tree development. $(\times 3.5$.
Axial pathways were chosen-that is, those passing to the distal pleural surface of a given segment-and the number of generations and the extension of glands and cartilage estimated (for discussion of methods and difficulties see Bucher and Reid, 1961a). Even in a normal lung there are variations between axial pathways within a segment, and, since with distorted lung the choice of the longest pathway might be difficult, several counts were made in each region to establish the maximum.

The amount of alveolar development was assessed by estimating the distance and the number of alveolar walls between the pleura and the terminal bronchioli lying close to it : this was done for the surface of the segment distant from the hilum and for the fissural surface. In eight separate sections of each lobe a count was made of the number of alveoli lying between the pleura and the nearest bronchiolus showing the transition from bronchiolar epithelium to alveoli. This distance was also measured in units of a graticule placed in the microscope eyepiece, which were converted into millimetres. In each case the mean of the eight counts was taken. Any variation between the lungs of the two infants are mainly the result of difference in antenatal development. as each child had breathed for only a couple of hours and in neither case had the lungs been inflated with formalin (fixation being achieved by immersion in formalin).

\section{Results}

Naked-eye Examination of Lungs. - These two cases of diaphragmatic hernia on naked-eye examination showed both right and left lungs to be reduced in volume, although a much greater reduction was seen on the left-the same as the hernia. The lobation was normal but not the shape of the lobes. In Case 2, in spite of their small size, the arrangement of the lobes was normal ; in Case 1, although the lobes were larger, the bronchi at the hilum were twisted, and it was only after reconstructing the hilar pattern of branching from serial sections that the lobes could be identified. On dissection it was found that the distal part of the left main bronchus received only the tip of a millimetre probe, while the diameter of the right main bronchus at this level was many times greater. In Case 2 the diameter of the left lower lobe bronchus was half that of the right lower lobe.

Examination of Serial Sections to Establish the Arrangement of the Intrasegmental Development.-In Table I normal and hypoplastic lungs are compared. In the normal, both upper and lower lobes show much the same range for maximum segmental counts. as each includes segments with a short axis as well as those with a long one : but both segments of the middle lobe and lingula are long, showing a narrow range of axial counts

TABLe I.-Maximum Counts Along Axial Pathways in Different Regions

\begin{tabular}{|c|c|c|c|c|c|c|}
\hline \multirow[b]{2}{*}{ Lobe } & & \multirow{2}{*}{$\begin{array}{c}\text { Normal } \\
\text { Range of } \\
\text { Maximum } \\
\text { Counts }\end{array}$} & \multicolumn{2}{|c|}{ Case 1} & \multicolumn{2}{|c|}{ Case 2} \\
\hline & & & $\begin{array}{l}\text { Left } \\
\text { Lung }\end{array}$ & $\begin{array}{l}\text { Right } \\
\text { Lung }\end{array}$ & $\begin{array}{l}\text { Left } \\
\text { Lung }\end{array}$ & $\begin{array}{l}\text { Right } \\
\text { Lung }\end{array}$ \\
\hline Upper &. & $17-20(13)$ & $9(7)$ & $13(8)$ & $\begin{array}{r}7(6) \\
6(3) \\
10(8)\end{array}$ & $14(8)$ \\
\hline $\begin{array}{l}\text { Middle or } \\
\text { lingula }\end{array}$ & •. & $22-26(16)$ & $12(8)$ & $\begin{array}{l}17(7) \\
16\end{array}$ & $\begin{array}{l}13(5) \\
14(8)\end{array}$ & $19(9)$ \\
\hline Lower & .. & $18-25(18)$ & $\begin{array}{l}16(9) \\
11(7)\end{array}$ & $\begin{array}{l}17(10) \\
14(10) \\
11(10)\end{array}$ & $\begin{array}{l}5(5) \\
9(9)\end{array}$ & \\
\hline
\end{tabular}

Figures in parentheses indicate generations with cartilage. 
(between 22 and 26). The extent of the cartilage along an axial pathway varies; sometimes half the pathways are free of cartilage, and therefore by definition bronchioli and at other times cartilage plates extend to within a few generations of the end of the axial pathway.

In no segment in these hypoplastic lungs were the counts of the branches within the normal range;. even further reduction was found in lateral pathways (not recorded in Table I). The left lung in each case shows a much greater reduction than the right, the left lung yielding counts which are mostly about half the normal, sometimes only a third, and less often as much as twothirds. The lowest count was in the lower lobe of Case 2. The right lung in both cases gave counts between onehalf and two-thirds of the normal. Although in Case 1 the child was more mature, the right lung was more affected than that of Case 2-which may reflect the size of the hernia and its relatively greater encroachment on the thoracic cage.

The bronchial and the bronchiolar region along a given pathway are both reduced. In many pathways in both these cases the proportion of bronchioli to bronchi would seem normal, but in other sites, such as the lower lobe of the left lung in Case 2, no bronchiolar region had developed, as alveoli opened directly from airways with cartilage in their wall-that is, bronchi (Fig. 2). In one axial pathway in the upper lobe only one generation was free of cartilage, which suggests that there again a relatively greater reduction had occurred in the bronchiolar than in the bronchial region of the lung; in other segments, notably the lingula, the opposite would seem to have been the case. Although arrest of development has occurred throughout both lungs, the changes are irregular in their distribution.

Development of Glands and Cartilage.-At birth mucous glands are present in only about one-third of generations along an axial pathway. The number of bronchi with glands is reduced in these hypoplastic lungs. Although the diameter of the left main bronchus was reduced in Case 1, the thickness of the individual plates of cartilage was roughly normal. In Case 2 the cartilage plates to the left lower lobe were only slightly reduced in thickness compared with the right.

Development of Alveoli.-The hypoplasia would seem to have affected the size of the alveoli rather than their number, the number of alveolar walls between a terminal bronchiolus and the pleura being mostly within the normal range (Emery and Mithal, 1960) although the distance was less (Table II).

TABLE II

\begin{tabular}{|c|c|c|c|c|c|}
\hline \multirow{2}{*}{\multicolumn{2}{|c|}{ Lobe }} & \multicolumn{2}{|c|}{ Case 1. Alveoli } & \multicolumn{2}{|c|}{ Case 2. Alveoli } \\
\hline & & No. & $\begin{array}{c}\text { Depth } \\
\text { (in mm.) }\end{array}$ & No. & $\underset{\text { (in } \mathrm{mm} \text {.) }}{\text { Depth }}$ \\
\hline Loft & $\begin{cases}\text { Upper } & \ldots \\
\text { Lower } & \ldots\end{cases}$ & $\begin{array}{l}3 \cdot 6 \\
2 \cdot 8\end{array}$ & $\begin{array}{l}0.12 \\
0.11\end{array}$ & $\begin{array}{l}4 \cdot 4 \\
4 \cdot 1\end{array}$ & $\begin{array}{l}0.23 \\
0.17\end{array}$ \\
\hline Right & $\left\{\begin{array}{l}\text { Upper } \\
\text { Middle } \\
\text { Lower }\end{array}\right.$ & $\begin{array}{l}4.5 \\
4.6 \\
4.5\end{array}$ & $\begin{array}{l}0.30 \\
0.25 \\
0.33\end{array}$ & $\begin{array}{l}4 \cdot 5 \\
4 \cdot 5\end{array}$ & $\begin{array}{l}0.27 \\
0.32\end{array}$ \\
\hline
\end{tabular}

\section{Discussion}

The disparity between the two cases suggests that both the size of the hernia and the maturity of the foetus affect alveolar development. Case 1 was the more mature of the two foetuses and the one with larger hernia, yet the left lung showed the greatest reduction in size and number of alveoli of the four lungs examined, the number of alveoli being below the normal range. In the right lung of both cases and, surprisingly, in the left lung of Case 2 , the alveolar count was roughly the same and within the normal range, although the depth of the alveolar layer-that is, the diameter of the alveoli-in the left lung of Case 2 was less than in the two right lungs. The fact that the size and number of the alveoli were less affected than the bronchial count may mean that, in the cases described here, alveolar development started rather earlier in intrauterine life than is normal.

In these two cases of congenital diphragmatic hernia not only were both lungs small but neither the right nor the left had its full complement of bronchial branches ; the reduction was greater on the side of the hernia, the count along an axial pathway being less than half the normal. Alveoli were rather less affected.

On the side of hernia the stage of bronchial arrest corresponded to an intrauterine development of 10 to 12 weeks, on the contralateral side to a development of 12 to 14 weeks. This suggests that at 10 weeks the abdominal contents were already above the diaphragm, although it cannot be assumed that they may not have entered the thoracic cage earlier. It has been suggested that herniation takes place at birth or soon after, but, at least in cases such as the two described here, the physical interference with the lung points to early herniation. If the smallness were due to compression, even in late foetal life, of a hitherto normally developed lung, the bronchial counts would not be reduced and it might be expected that the lung would expand more easily at operation to fill the chest than in fact it does.

This invasion of the thorax affects both lungs, the ipsilateral more than the contralateral, and on the side of the hernia the lower lobe more than the upper. Campanale and Rowland (1955) have shown that the heart is displaced, thereby encroaching on the opposite hemithorax and rendering the contralateral lung also hypoplastic. The result for both lungs is reduction of space.

Bronchial branching normally continues until the fourteenth or sixteenth weeks of intrauterine life, the duration varying from segment to segment according to the number of branches along a given pathway. In the present cases the number of generations corresponded to 10 or 12 weeks' normal gestation, for the reason either that growth continued for the normal time but was slower or that it ceased prematurely. The latter hypothesis is supported by the fact that the normal alveolar level is achieved even in the case of the premature baby.

The fact that these small lungs have differentiated to the alveolar phase from the glandular and that the number of alveoli found distal to a terminal bronchiolus is normal means that correction of the hernia may lead to aeration and growth of the hypoplastic lung, resulting in the total normal alveolar endowment. Beyond the occasional report that within a few months of operation the radiograph may appear " normal," little is known of the long-term behaviour of these lungs.

For an indication of the possible behaviour of these lungs it is necessary to turn to animal experiments which point the importance of the space available to the lung for normal growth as well as growth compensatory to disease. Rienhoff, Reichert, and Heuer (1935) found that in dogs, after pneumonectomy, an increase in size of the residual lung occurred by the development of new alveoli but not from the formation of new bronchiolar 
generations. Cohn (1939), by assessing the weight of the exsanguinated lobes of rats, followed the effect on growth of various procedures which altered volume; he found that the weight of residual lung increased rapidly after resection and, conversely, that the normal age increase in weight did not occur if the volume of the thoracic cage was reduced by a pneumoperitoneum. It would seem that in human lungs also little if any increase in bronchiolar generations normally occurs after birth (Boyden, 1961, 1962; Reid, 1961)-that growth is distal to the terminal bronchiolus within the acinus. In hypoplastic lungs the number of terminal bronchioli and the volume of alveolar tissue are reduced. It would be expected that no correction of the bronchial and bronchiolar deficiency will occur, but that growth within the acini will be great enough to provide the lung with its normal complement of alveoli.

\section{Summary}

The features of the hypoplasia associated with congenital diaphragmatic hernia have been studied by serial section of the lungs from two newborn infants with congenital diaphragmatic hernia. The number of bronchial branchings was greatly reduced in all four lungs, the effect being greater in the ipsilateral lungs, with axial pathways giving counts of half the normal values or less, while in the contralateral lungs the count was rather more than half of the normal. Bronchial and bronchiolar regions were both affected : on occasion the bronchiolar region was completely lacking. Thus the brunt of the change falls on the bronchial tree, as the alveoli are relatively less affected, in three of the four lungs the counts being within the normal range.

The bronchial arrest is at a stage normally achieved at 10 to $12 \frac{1}{2}$ weeks, suggesting that at this time abdominal contents had already invaded the thorax. The arrest of bronchial branching which occurs during the "glandular" phase may be responsible for premature differentiation to the "alveolar" stage.

The nature of growth following surgical correction of the hernia is discussed in the light of experimental and anatomical studies.

\section{REFERENCES}

Barrett. N. R., and Wheaton, C. E. W. (1934). Brit. J. Surg., 21420.

Boyden. E. A. (1961). Personal communication.

- (1962). J. thorac Surg. In press.

Bucher, U., and Reid, L. (1961a). Thorax, 16, 207.

- (1961b). Ibid., 16. 219.

Campanale, R. P., and Rowland, R. H. (1955). Ann. Surg., 142, 176.

Cohn, R. (1939). Anat. Rec., 75, 195. Emery, J. L., and Mithal, A. (1960). Arch. Dis. Childh., 35,

Potter, E. L. (1962). Pathology of the Fetus and the Newborn, 2nd ed., p. 301. Year Book Publishers, Chicago.

Reid, L. (1961). Bronches. 11, 573.

Rienhoff, W. F., Reichert, F. L., and Heuer, G. J. (1935). Johns Hopk. Hosp. Bull.. 57, 373.

Tolins, S H. (1953). Ann. Surg., 137, 276.

"A painting portraying an action during the Basuto rebellion of 1880-1 was presented to the Royal Army Medical College in Millbank, S.W. [on December 14]. by the South African Ambassador in London. Dr. H. Muller. The action portrayed in the painting was one in which Surgeon Major John Frederick McCrea won the Victoria Cross at Tweefontein in 1881. The painting will hang in the V.C. room at Millbank, along with other Victoria Cross paintings." (The Times, December 15.)

\section{CARCINOMA OF BRONCHUS WITH HYPONATRAEMIA AND DERMATOMYOSITIS}

BY

\author{
R. T. WILLIAMS, M.B., M.R.C.P. \\ Lecturer in Medicine, University of Manchester
}

Schwartz et al. (1957) described the cases of two patients with carcinoma of the bronchus who developed hyponatraemia which was unlike the hyponatraemia associated with states of sodium depletion. There was no evidence of renal or adrenal disease, and yet, in spite of extreme hypotonicity of the serum, the urine remained hypertonic to serum. From the resemblance of this picture to that seen in normal subjects under the continuous influence of posterior pituitary extract it was postulated that these patients were secreting antidiuretic hormone (A.D.H.) continuously: the low serum sodium is not due to excessive loss of sodium but to abnormal retention of water. Since 1957 further reports of the syndrome in association with carcinoma of the bronchus have appeared (Roberts, 1959 : Schwartz et al., 1960 : Dossetor et al., 1961: Ivy, 1961: Turner and Williams, 1962), and the same mechanism has been postulated in central nervous system disease (Golberg and Handler, 1960 ; Carter et al., 1961 : Epstein et al., 1961 ; Van 't Hoff and Zilva, 1961). The syndrome has also been reported in a patient without evidence of other disease (Grumer et al., 1962).

Dermatomyositis of ten occurs with malignant disease, the association varying from $9.2 \%$ to $17.7 \%$ in the major series of dermatomyositis that have been reported (Schuermann, 1951 : Curtis et al., 1952; Walton and Adams, 1958 : Pearson and Rose, 1960). The neoplasm may be of any type, but bronchial, ovarian, mammary, and gastric carcinomas figure largely.

This report concerns a man with bronchial carcinoma who developed both hyponatraemia and dermatomyositis.

\section{Case Report}

A 44-year-old man was admitted to Manchester Royal Infirmary on October 4. 1960. For three months be had noticed increasing breathlessness on exertion and a cough with purulent sputum. Chest radiographs showed a large left pleural effusion and a mass at the left hilum, and at bronchoscopy (Mr. D. T. Thompson) the left main bronchus was found to be occupied by a fleshy nodular tumour which proved to be an oat-cell carcinoma. Because the pleural effusion reaccumulated after aspiration it was decided that surgical treatment was not feasible and he was treated with mustine hydrochloride (nitrogen mustard). After three injections of trimustine hydrochloride ("trillekamin ") the pleural effusion did not recur and he was discharged on November 11.

One week before his second admission. on January 24, 1961, he developed an erythematous rash over the butterfly area of his face, and this rash persisted. On February 21 he first complained of aching and tenderness in the muscles of the upper arms, particularly the deltoids, and over the next few days he developed oedema of his face and arms. The proximal muscles of his arms became increasingly weak, so that he had the greatest difficulty in lifting either hand to his mouth and found it impossible to brush his hair. He also complained of dysphagia, and this rapidly progressed to a stage where he had to subsist on semi-solid foods and liquids. His voice was hoarse. but the vocal cords were fully mobile. It was now apparent that he was suffering from acute dermatomyositis, and in addition to the above features 